\title{
Supplementary Material for \\ Lookup Peaks: A More Sensitive Hybrid of De Novo Sequencing and Database Search for Protein Identification by Tandem Mass Spectrometry
}

\author{
Marshall Bern* $\quad$ Yuhan Cai ${ }^{\dagger} \quad$ David Goldberg*
}

\section{Algorithmic Details}

Here we give sufficient details of the algorithms in ByOnic to allow any skilled programmer to reproduce the tool. An observed spectrum is a "centroided" spectrum, in .dta format, as output by instrument software such as XCalibur. Such a spectrum consists of peaks $p$, each of which is a pair of real numbers: a mass-over-charge $m(p)$ and an intensity $I(p)$. In addition, an observed spectrum includes a parent mass (or mass-over-charge) and a charge assignment, typically between +1 and +4 . ByOnic is really a pipeline of three programs, called ByPair, LookUp, and MutScore.

\subsection{Computing Lookup Peaks}

The first program, ByPair, starts by manipulating peak intensities. Intensity is first changed to "local intensity", a measure of the strength of a peak $p$ relative to other nearby peaks, by replacing $I(p)$ by the following expression:

$$
\frac{I(p)}{\sum_{p^{\prime}} I\left(p^{\prime}\right) /\left|m(p)-m\left(p^{\prime}\right)\right|}
$$

To avoid comparing a peak against other peaks in the same isotopic series, the sum in the denominator is over $p^{\prime}$ such that $\left|m(p)-m\left(p^{\prime}\right)\right|>3.2$. Next intensities are modified to reflect isotopic series. If peak $p$ is accompanied by a peak $p^{\prime}$ of the right mass-over-charge (for example, $m(p)+0.7<$ $m\left(p^{\prime}\right)<m(p)+1.3$ for ion-trap instruments) and the appropriate intensity (for example, $0.4 \cdot I(p)<$ $I\left(p^{\prime}\right)<1.2 \cdot I(p)$ for $\left.800 \leq m(p)<1200\right)$, then peak $p$ is upgraded by multiplying $I(p)$ by 1.2 and peak $p^{\prime}$ is downgraded by multiplying $I\left(p^{\prime}\right)$ by 0.2 . The intensities at this point are the modified intensities that are used by the scorer.

For computing lookup peaks, however, we perform further manipulations. If peak $p$ is accompanied by a peak $p^{\prime}$ such that $m(p)-18.4<m\left(p^{\prime}\right)<m(p)-17.6$, then we multiply $I(p)$ by 1.5 and $I\left(p^{\prime}\right)$ by 0.5 , because $p^{\prime}$ is a likely water-loss peak. If the user has set a flag that tells ByPair to trust the parent ion mass, then ByPair boosts the intensities of both peaks (multiplying by 1.5) in each pair that sums to the parent mass (within a tolerance), as these peaks are likely complementary ions from the same cleavage.

ByPair then sorts the peaks by decreasing intensity $I(p)$. Starting from the most intense peak, each peak $p$ next propagates intensity to other peaks, by replacing $I\left(p^{\prime}\right)$ by $\left(I\left(p^{\prime}\right)+I(p)\right) / 2$ for each peak $p^{\prime}$ such that $I\left(p^{\prime}\right)<I(p)$, and $\left|m(p)-m\left(p^{\prime}\right)\right|$ is within the mass tolerance of an amino acid residue mass. The rationale here is that $\mathrm{b}$ - and y-ion peaks connect to other $\mathrm{b}$ - and y-ion peaks, but noise peaks are unlikely to have such connections. ByPair performs two propagation cycles.

*Palo Alto Research Center, 3333 Coyote Hill Rd., Palo Alto, CA 94304 \{bern, goldberg\}@parc.com

${ }^{\dagger}$ Computer Science and Engineering, U. of Washington, Seattle, WA 98195 yuhancai@cs.washington. edu 


\begin{tabular}{|l|rrrrr|rrrrrr|rrrr|}
\hline \multicolumn{1}{|c|}{ \# Lookup Pairs: } & \multicolumn{4}{|c|}{ 10 Pairs } & \multicolumn{4}{c|}{ 14 Pairs } & \multicolumn{4}{c|}{ 20 Pairs } \\
\hline \multicolumn{1}{|c}{ \# Correct: } & 0 & 1 & 2 & 3 & $\geq 4$ & 0 & 1 & 2 & 3 & $\geq 4$ & 0 & 1 & 2 & 3 & $\geq 4$ \\
\hline Full Pairs & 11 & 15 & 27 & 29 & 223 & 7 & 12 & 25 & 23 & 238 & 5 & 11 & 21 & 26 & 242 \\
Individual Peaks & 0 & 3 & 8 & 6 & 288 & 0 & 2 & 3 & 6 & 294 & 0 & 0 & 2 & 5 & 298 \\
\hline
\end{tabular}

Table 1. This table gives the number of spectra, within a training set of 305 well-identified ion-trap spectra, with $0,1,2,3$, or at least 4 correct lookup pairs. For example, when using 10 lookup pairs, 11 spectra had no correct pairs, 15 had only one correct pair, 27 had two, 29 had three, and 223 had four or more. Similarly, 0 spectra had no correct individual peaks within these pairs, 3 had only one correct peak, and so forth. Notice that correct individual peaks (flanking masses without tags) are somewhat easier to compute than correct pairs (length-1 tags).

ByPair then forms peak pairs $\left(p, p^{\prime}\right)$. Each peak pair is such that $m\left(p^{\prime}\right)-m(p)$ is within the mass tolerance of an amino acid residue mass. The intensity assigned to pair $\left(p, p^{\prime}\right)$ is $\min \left\{I(p), I\left(p^{\prime}\right)\right\}$. ByPair then sorts peak pairs by decreasing intensity and chooses output pairs from the beginning of the list, until the desired number of pairs (typically 14) has been found. If $\left(p, p^{\prime}\right)$ is chosen as an output pair, the numbers actually emitted by ByPair are $i(p)$ and $i\left(p^{\prime}\right)$, where $i(p)$ is the closest integer to $0.9995 \cdot m(p)$. Each peak can appear in the same position (first or second) in an output pair at most twice. Thus if we have already chosen $(p, q)$ and $(p, r)$, we simply skip over $(p, s)$. The lookup peaks are the integers appearing in at least one output pair. If there are 14 pairs, then there are typically 15 to 25 lookup peaks. If the user has set the flag that tells the program to trust the parent ion mass $M$, then the program also outputs $M-i\left(p^{\prime}\right)$ and $M-i(p)$ if it outputs $i(p)$ and $i\left(p^{\prime}\right)$. As seen in Table 1, the number of correct individual peaks and peak pairs improves as the number of generated pairs grows. The filtering factor, however, degrades if the number of pairs grows without changing the hit requirements. We judged 14 pairs to be about the right balance. Using 14 pairs along with their complements does not degrade the filtering factor as much as using 28 independent pairs.

The second program in the ByOnic pipeline, called LookUp, uses the output of ByPair to find candidates in the database. A peptide in the database is selected as a candidate if it meets three requirements: (1) its mass (or the mass of an anticipated modification of the peptide) must match the spectrum parent mass within a user-specified tolerance (typically $[-C-2, C+1]$ where $C$ is the parent ion charge); (2) its computed b- and y-ions must match at least a certain number of lookup peaks; (3) its successive pairs of $b$ - and y-ions must match at least a certain number of output pairs. Requirements (2) and (3) are somewhat redundant, but LookUp allows both options for greater control of the amount of filtering. The required numbers of matched peaks and pairs can be set separately for tryptic, semi-tryptic, and non-tryptic peptides.

Figure 1 studies the identification rate as a function of the amount of filtering for data sets of iontrap spectra from two samples of reference proteins: 1124 spectra from the ISB data set as described in the text, and 3557 spectra from the Yates laboratory (The Scripps Research Institute), digested by a cocktail of four different proteases (described by MacCoss et al., Anal. Chem. 74 (2002), 55935599). Filtering factor is defined to be the ratio of the total number of candidates that satisfied the parent mass requirement (1) to the number that also satisfied the lookup-peak requirements (2) and (3). For the tryptic sample, we considered only unmodified, fully tryptic peptides and varied the filtering factor by requiring 1 matched peak out of 14 pairs, 2 out of 20, 2 out of 14, 3 out of 20, 3 out of 14, 4 out of 20, and 4 out of 14 . (The leftmost point on the curve shows a run with no filtering.) For the non-tryptic sample, we considered all unmodified peptides and varied the filtering factor by requiring 1 peak out of 14 pairs, 2 peaks out of 20, 1 pair or 3 peaks out of 20, 1 pair or 3 peaks out of 14,2 pairs or 4 peaks out of 20 , and so forth. 


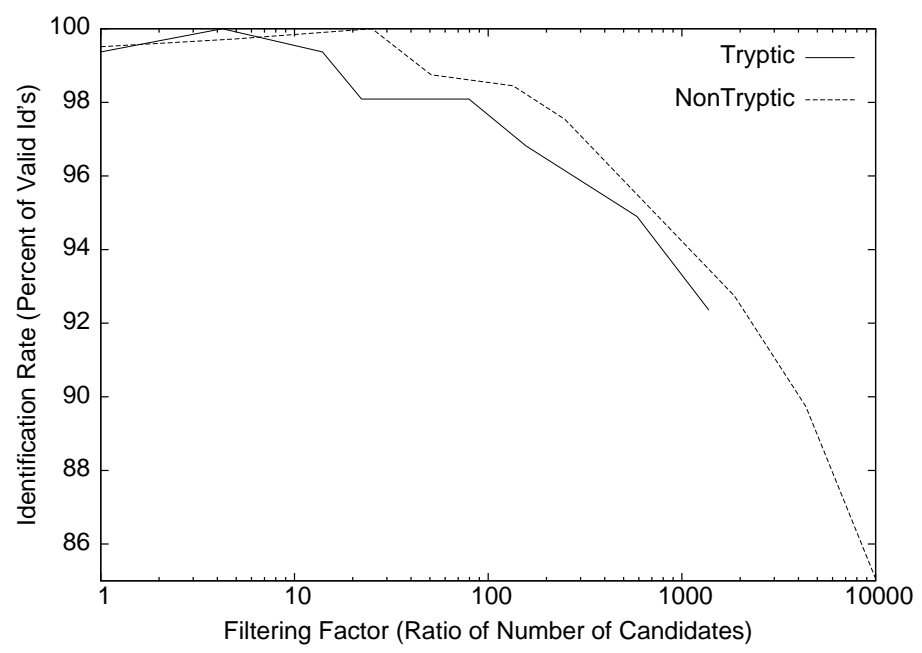

Figure 1. The number of valid identifications versus the filtering factor. A small amount of filtering actually increased the number of valid identifications slightly, by reducing the number of decoys.

\subsection{Scoring}

The final program in the pipeline is called MutScore. MutScore's score is simply a sum of benefits and penalties. The benefits accrue from matches between observed peaks and theoretical peaks. An observed peak matches at most one theoretical peak, and vice versa. For simplicity, matches are independent. A slight improvement in scoring accuracy, however, can be obtained by allowing dependences, for example, not scoring $b_{5}-18$ (a water-loss peak) unless $b_{5}$ is also observed. Dependencies slow down the scoring somewhat, so we did not use them in MutScore.

The benefit for matching an observed peak $p$ with a theoretical peak $p^{\prime}$ is the product $W(p)$. $A\left(p, p^{\prime}\right) \cdot E\left(p^{\prime}\right)$, where $W(p)$ is a function of the modified intensity of $p$ in the observed spectrum (Figure 2), $A\left(p, p^{\prime}\right)$ is a function of the mass accuracy of the match (Figure 3), and $E\left(p^{\prime}\right)$ is the theoretically expected intensity. Theoretically expected intensities were set as rough compromises between published statistics (Refs. 25 and 26 in the article) on b- and y-ion intensities, and statistics we gathered on training data for $\mathrm{b}$ - and y-ions, along with water and ammonia losses.

For a peptide of length $n$, the values for $E\left(p^{\prime}\right)$ are as follows: for the $b_{1}$ ion $E\left(p^{\prime}\right)=0.5$; for $b_{2}$ it is 1.2 ; for $b_{3}$ through $b_{k}$, where $k=\min \{n / 3,8\}$, it is 1.4 ; for $b_{k+1}$ through $b_{12}$ it is 1.6 ; for $b_{13}$ through $b_{15}$ it is 1.2 ; and for $b_{16}$ or larger it is 1.0. If the succeeding amino acid residue is proline, then $E\left(p^{\prime}\right)$ is multiplied by 0.6 , and if the preceding amino acid residue is proline, then $E\left(p^{\prime}\right)$ is multiplied by 1.4. Water losses from $\mathrm{b}$-ions containing $\mathrm{S}, \mathrm{T}, \mathrm{D}$, or $\mathrm{E}$ have weight 0.7 times the weight of the b-ion itself; ammonia losses from b-ions containing $\mathrm{N}, \mathrm{Q}, \mathrm{K}$, or $\mathrm{R}$ have weight 0.3 times the weight of the b-ion; carbon monoxide losses (a-ions) are weighted 0.3 times the weight of the $\mathrm{b}$-ion for $b_{1}$ through $b_{5}$ and 0.2 times for $b_{6}$ through $b_{8}$. If the b-ion peptide includes a phosphorylation then the phosphate loss $(-98)$ is weighted 0.5 times the weight of the b-ion. For the $y_{1}$ and $y_{2}$ ion $E\left(p^{\prime}\right)=$ 1.2; for $y_{3}$ it is 1.5 ; for $y_{4}$ it is 1.7 ; for $y_{5}$ through $y_{k}$ it is 2.0 , where $k=\min \{n-3,13\}$; for $y_{k+1}$ through $y_{n-1}$ it is 1.5 . Proline modifies $E\left(p^{\prime}\right)$ for y-ions just as it does for b-ions. Water losses have weight 0.4 times the weight of the y-ion; ammonia losses have weight 0.2 times the weight of the $\mathrm{y}$-ion; and phosphate losses have weight 0.5 times the weight of the y-ion. All doubly charged y-ions with mass at least 900 Daltons have $E\left(p^{\prime}\right)=0.5$ if the parent charge is 2, and have $E\left(p^{\prime}\right)=1.0$ if the parent charge is 3 or greater.

There is an additional benefit for matched peaks, regardless of match accuracies and theoreti- 


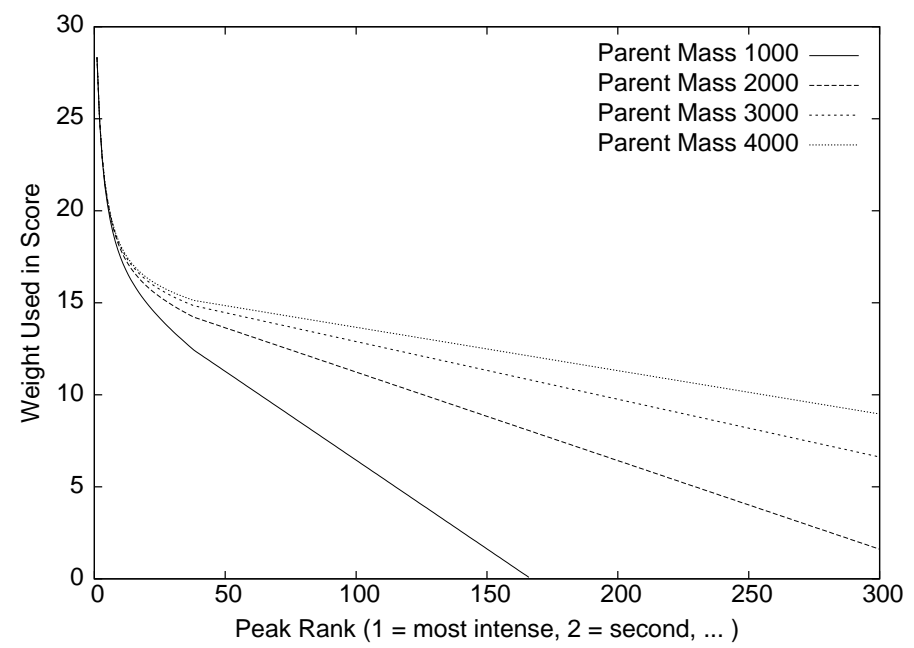

Figure 2. The weight $W(p)$ of a matched observed peak $p$ depends upon its modified-intensity rank and the parent mass of the peptide being scored. Larger parent ions generate more b- and y-ion peaks, so MutScore counts lower rank peaks more heavily. In any case, MutScore scores at most 300 peaks per spectrum.

cal intensities. We add to MutScore's score the percent of the total spectral intensity (unmodified intensity) included in the matched observed peaks; this benefit naturally runs from 0 to 100 .

MutScore subtracts a penalty for each theoretical peak $p^{\prime}$ not matched. The penalty is $E\left(p^{\prime}\right)$ for all theoretical peaks with mass-over-charge in the ranges of [200,500] and [1500,2000] Thompsons (Daltons divided by charge), and is $2 \cdot E\left(p^{\prime}\right)$ for all those with mass-over-charge between 500 and 1500. In addition, for tryptic digests, MutScore subtracts 10.0 for each non-tryptic terminus, and subtracts 5.0 for each internal $\mathrm{R}$ or $\mathrm{K}$.

MutScore provides a menu of modifications, shown in Table 2. Modifications do not change $E\left(p^{\prime}\right)$, with the one exception that phosphorylation allows loss of phosphate. It is known that oxidized methionine has a neutral loss of -64 , but this is not currently included in MutScore. MutScore has various pre-set scoring modes: (1) no-modifications search; (2) common-modifications search, which allows three oxidations ( $\mathrm{M}, \mathrm{H}$, and $\mathrm{W}$ ), the two pyro-glus, the two deamidations, and hydroxyproline; (3) all-modifications search, which allows all modifications but does not search for multiple phosphorylations; (4) phosphorylation search, which allows only the phosphorylation modifications shown in the last two lines of the table, but up to any combination of three modifications; (5) mutation search, any one-residue mutation; and (6) blind modification search, any integer change to any one residue, within a range such as plus or minus 100 Daltons.

\section{Experiments}

Table 3 summarizes the results of the experiments on data set (2), the 1200 QTOF spectra of human blood plasma. Table 4 gives the 13 human proteins spiked into mouse blood plasma for data set (3). 


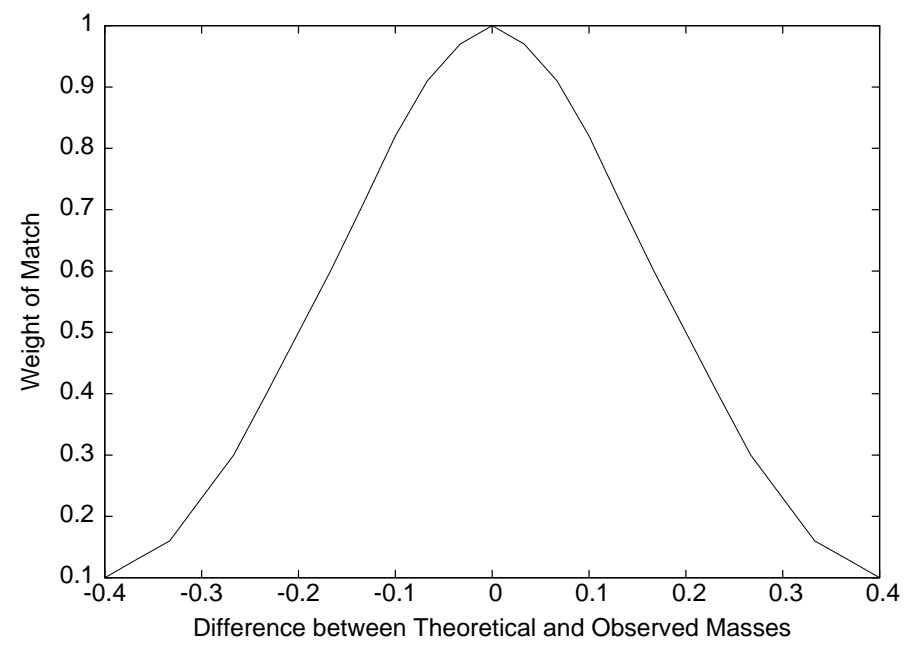

Figure 3. The term $A\left(p, p^{\prime}\right)$ weights a match of an observed peak $p$ and a theoretical peak $p^{\prime}$ according to the mass error of the match. MutScore uses the same curve for all mass spectrometers, with the $\mathrm{x}$-axis scaled by the user-set tolerance, typically 0.4 for ion-trap instruments, as shown above, and 0.08 for QTOF.

\begin{tabular}{|l|l|r|l|}
\hline Name & Amino Acid Residues & $\Delta$ Mass & Occurrence \\
\hline Oxidation & M H W Y & +15.995 & Artifact in vitro, Disease process in vivo \\
Deamidation & N Q & +0.984 & Artifact \\
Pyro-glu from E & N-terminal E & -18.011 & Artifact \\
Pyro-glu from Q & N-terminal Q & -17.027 & Artifact \\
Sodiation & E D Peptide C-terminus & +21.982 & Artifact \\
Carbamylation & R K Peptide N-terminus & +43.006 & Artifact \\
Dehydration & D & -18.011 & Artifact \\
Disulfide bridge & C..C & -2.016 & Artifact with untreated cysteine \\
Carboxymethylation & N-terminus & +58.005 & Artifact with iodoacetic acid treatment \\
Carboxylation & M & +43.990 & Artifact with iodoacetic acid treatment \\
Amidation & Protein C-terminus & -0.984 & Post-translational modification \\
Methylation & K R H I L N Q Protein N-terminus & +14.016 & Post-translational modification \\
Dimethylation & K R N Protein N-terminus & +28.031 & Post-translational modification \\
Acetylation & C S K Protein N-terminus & +42.011 & Post-translational modification \\
Hydroxylation & P D K N F R & +15.995 & Post-translational modification \\
Phosphorylation & S T Y & +79.966 & Post-translational modification \\
Loss of Phosphate & S T & -18.011 & Post-translational mod plus neutral loss \\
\hline
\end{tabular}

Table 2. MutScore currently supports the variable modifications above, along with the standard fixed modifications of cysteine (every $\mathrm{C}$ is assumed to be so modified): carbamidomethylation $(+57.051)$ and carboxymethylation $(+58.005)$. 


\begin{tabular}{|c|c|c|c|c|c|}
\hline Rank & Protein Description & ByOnic & $\%$ Cover & Mascot & $\mathrm{X}$ !Tandem \\
\hline 1 & Alpha-2-macroglobulin precursor (Alpha-2-M) & 154 & 68.38 & 106 & 120 \\
\hline 2 & Complement $\mathrm{C} 3$ precursor & 135 & 60.25 & 93 & 119 \\
\hline 3 & Apolipoprotein B-100 precursor (Apo B-100) & 100 & 21.48 & 66 & 86 \\
\hline 4 & Ceruloplasmin precursor (EC 1.16.3.1) (Ferroxidase) & 44 & 40.28 & 36 & 38 \\
\hline 5 & Apolipoprotein A-I precursor (Apo-AI) (ApoA-I) & 73 & 77.53 & 42 & 56 \\
\hline 6 & Hemopexin precursor (Beta-1B-glycoprotein) & 37 & 56.28 & 31 & 31 \\
\hline 7 & Vitamin D-binding protein precursor (DBP) & 21 & 29.32 & 16 & 18 \\
\hline 8 & Alpha-1-antichymotrypsin precursor (ACT) & 20 & 46.81 & 13 & 18 \\
\hline 9 & Apolipoprotein A-II precursor (Apo-AII) (ApoA-II) & 30 & 69.99 & 20 & 26 \\
\hline 10 & Inter-alpha-trypsin inhibitor heavy chain $\mathrm{H} 2$ precursor & 21 & 23.15 & 14 & 15 \\
\hline 11 & Alpha-1B-glycoprotein precursor (Alpha-1-B glycoprotein) & 20 & 34.95 & 11 & 16 \\
\hline 12 & Beta-2-glycoprotein I precursor (Apolipoprotein $\mathrm{H})($ Apo-H) & 14 & 41.45 & 13 & 11 \\
\hline 13 & Complement $\mathrm{C} 4$ precursor $(\mathrm{C} 4-\mathrm{A}$ and $\mathrm{C} 4-\mathrm{B})$ & 55 & 29.99 & 41 & 43 \\
\hline 14 & Complement factor B precursor (EC 3.4.21.47) & 16 & 21.33 & 12 & 13 \\
\hline 15 & Alpha-1-acid glycoprotein 1 precursor (AGP 1) (Orosomucoid-1) & 17 & 44.77 & 9 & 14 \\
\hline 16 & Angiotensinogen precursor & 10 & 21.65 & 9 & 10 \\
\hline 17 & Inter-alpha-trypsin inhibitor heavy chain $\mathrm{H} 1$ precursor & 10 & 14.82 & 9 & 7 \\
\hline 18 & Antithrombin-III precursor (ATIII) & 21 & 37.28 & 19 & 18 \\
\hline 19 & Kininogen-1 precursor (Alpha-2-thiol proteinase inhibitor) & 10 & 15.22 & 11 & 9 \\
\hline 20 & Haptoglobin precursor & 19 & 38.92 & 16 & 18 \\
\hline 21 & Alpha-2-HS-glycoprotein precursor (Fetuin-A) & 16 & 41.42 & 8 & 11 \\
\hline 22 & Apolipoprotein A-IV precursor (Apo-AIV) (ApoA-IV) & 10 & 27.52 & 8 & 9 \\
\hline 23 & Plasma protease $\mathrm{C} 1$ inhibitor precursor (C1 Inh) (C1Inh) & 9 & 18.40 & 9 & 8 \\
\hline 24 & Inter-alpha-trypsin inhibitor heavy chain $\mathrm{H} 4$ precursor & 9 & 8.49 & 7 & 10 \\
\hline 25 & Histidine-rich glycoprotein precursor & 5 & 11.05 & 5 & 5 \\
\hline 26 & Heparin cofactor II precursor (HC-II) & 9 & 15.43 & 8 & 8 \\
\hline 27 & Vitronectin precursor (Serum spreading factor) & 9 & 17.78 & 5 & 7 \\
\hline 28 & AMBP protein precursor & 5 & 14.20 & 4 & 4 \\
\hline 29 & Alpha-1-acid glycoprotein 2 precursor (AGP 2) (Orosomucoid-2) & 5 & 34.33 & 6 & 3 \\
\hline 30 & $\mathrm{C} 4 \mathrm{~b}$-binding protein alpha chain precursor $(\mathrm{C} 4 \mathrm{bp})$ & 5 & 8.54 & 6 & 6 \\
\hline 31 & Apolipoprotein C-III precursor (Apo-CIII) & 6 & 45.45 & 4 & 4 \\
\hline 32 & Plasminogen precursor (EC 3.4.21.7) & 13 & 16.30 & 5 & 8 \\
\hline 33 & Serotransferrin precursor (Transferrin) (Siderophilin) & 4 & 8.02 & 4 & 3 \\
\hline 34 & Complement factor $\mathrm{H}$ precursor $(\mathrm{H}$ factor 1$)$ & 6 & 5.69 & 4 & 4 \\
\hline 35 & Prothrombin precursor (EC 3.4.21.5) (Coagulation factor II) & 5 & 7.88 & 4 & 5 \\
\hline 36 & Ig alpha- 1 chain $\mathrm{C}$ region & 5 & 18.13 & 2 & 5 \\
\hline 37 & Ig lambda chain $\mathrm{C}$ regions & 2 & 28.57 & 2 & 2 \\
\hline 38 & Ig mu chain $\mathrm{C}$ region & 7 & 15.64 & 6 & 5 \\
\hline 39 & Apolipoprotein E precursor (Apo-E) & 5 & 17.03 & 4 & 5 \\
\hline 40 & Clusterin precursor (Complement-associated protein SP-40,40) & 3 & 7.35 & 2 & 2 \\
\hline 41 & Plasma retinol-binding protein precursor (PRBP) & 3 & 8.95 & 2 & 2 \\
\hline 42 & Serum paraoxonase/arylesterase 1 (EC 3.1.1.2) & 3 & 9.32 & 2 & 2 \\
\hline 43 & Apolipoprotein C-I precursor (Apo-CI) (ApoC-I) & 3 & 33.73 & 2 & 1 \\
\hline 44 & Ig gamma- 3 chain $\mathrm{C}$ region (Heavy chain disease protein) & 3 & 10.00 & 1 & 2 \\
\hline 45 & Ig kappa chain $\mathrm{C}$ region & 2 & 15.09 & 2 & 3 \\
\hline 46 & $\mathrm{~N}$-acetylmuramoyl-L-alanine amidase precursor (EC 3.5.1.28) & 3 & 6.42 & 1 & 0 \\
\hline 47 & Complement component $\mathrm{C} 8$ alpha chain precursor & 3 & 5.65 & 1 & 1 \\
\hline 48 & Complement C1r subcomponent precursor (EC 3.4.21.41) & 2 & 3.97 & 1 & 1 \\
\hline 49 & Transthyretin precursor (Prealbumin) (TBPA) (TTR) & 1 & 14.96 & 1 & 2 \\
\hline 50 & Ficolin-3 precursor & 1 & 4.68 & 1 & 1 \\
\hline 51 & Complement factor I precursor (EC 3.4.21.45) & 1 & 2.23 & 1 & 1 \\
\hline 52 & Apolipoprotein C-II precursor (Apo-CII) & 3 & 29.70 & 2 & 2 \\
\hline 53 & Apolipoprotein M (Apo-M) (ApoM) (G3a protein) & 2 & 10.11 & 1 & 0 \\
\hline 54 & Complement component $\mathrm{C} 9$ precursor & 2 & 3.40 & 0 & 1 \\
\hline 55 & Complement C5 precursor & 3 & 1.31 & 2 & 3 \\
\hline 56 & Serum amyloid P-component precursor (SAP) & 2 & 6.28 & 2 & 3 \\
\hline 57 & Alpha-2-antiplasmin precursor (Alpha-2-plasmin inhibitor) & 2 & 4.89 & 2 & 2 \\
\hline 58 & Gelsolin precursor (Actin-depolymerizing factor) (ADF) (Brevin) & 2 & 1.53 & 2 & 1 \\
\hline 59 & Afamin & 2 & 1.53 & 2 & 2 \\
\hline 60 & Fibulin-1 precursor & 2 & 1.85 & 0 & 0 \\
\hline
\end{tabular}

Table 3. The top blood plasma proteins in the 1200 QTOF spectra. The columns labeled "ByOnic", "Mascot", and "X!Tandem" give the number of spectra each tool matched to each protein; "Cover" gives ByOnic's percent coverage. 


\begin{tabular}{|l|l|}
\hline Accession Number and Gene & Protein Description \\
\hline gi 1082350 pir B55053 HUMAN & Endothelial monocyte-activating protein II precursor \\
gi 5730097 ref NP-006564.1 & Tumor necrosis factor 13b \\
gi6015145 sp O43320 FGF16-HUMAN & Fibroblast growth factor 16 (FGF-16) \\
gi 6015147 sp O60258 FGFH-HUMAN & Fibroblast growth factor-17 precursor (FGF-17) \\
gi 730218 sp P41159 OB-HUMAN & Leptin precursor (Obesity factor) \\
gi 11132196 sp O95750 FGFJ-HUMAN & Fibroblast growth factor-19 precursor (FGF-19) \\
gi 113992 sp P02647 APA1-HUMAN & Apolipoprotein A-I precursor (Apo-AI) \\
gi 122750 sp P08620 FGF4-HUMAN & Fibroblast growth factor-4 precursor (FGF-4) (HST-1) \\
gi 130930 sp P01236 PRL-HUMAN & Prolactin precursor (PRL) \\
gi 135934 sp P01375 TNFA-HUMAN & Tumor necrosis factor precursor (TNF-alpha) (TNF-a) (Cachectin) \\
gi 13637763 sp P12034 FGF5-HUMAN & Fibroblast growth factor-5 precursor (FGF-5) (HBGF-5) \\
gi 21264084 sp O00300 TR11B-HUMAN & Tumor necrosis factor 11B precursor (Osteoprotegerin) \\
gi 4507609 ref NP-003802.1 HUMAN & Tumor necrosis factor 9 \\
\hline
\end{tabular}

Table 4. The 13 human proteins spiked into mouse blood plasma for data set (3). 